\title{
GENERALIZED VECTOR QUASI-VARIATIONAL-LIKE INEQUALITIES
}

\author{
JIAN-WEN PENG AND XIN-MIN YANG
}

Received 14 September 2004; Revised 11 November 2004; Accepted 17 November 2004

Using maximal element theorem, we prove some existence theorems for the two types of generalized vector quasi-variational-like inequalities with non-monotonicity and noncompactness.

Copyright (c) 2006 J.-W. Peng and X.-M. Yang. This is an open access article distributed under the Creative Commons Attribution License, which permits unrestricted use, distribution, and reproduction in any medium, provided the original work is properly cited.

\section{Introduction and preliminaries}

Let $Y$ be a real Hausdorff topological vector space and $X$ be a nonempty convex subset in a real locally convex Hausdorff topological vector space $E$. We denote $L(E, Y)$ the space of all continuous linear operators from $E$ into $Y$ and by $\langle u, y\rangle$ the evaluation of $u \in L(E, Y)$ at $y \in E$. Let $\sigma$ be the family of all bounded subsets of $X$ whose union is total in $E$, that is, the linear hull of $\cup\{S: S \in \sigma\}$ is dense in $X$. Let $\beta$ be a neighbourhood base of 0 in $Y$. When $S$ runs through $\sigma, V$ through $\beta$, the family

$$
M(S, V)=\left\{l \in L(E, Y): \cup_{x \in S}\langle l, x\rangle \subset V\right\}
$$

is a neighbourhood base of 0 in $L(E, Y)$ at $x \in E$ (see [29, pages 79-80]). By the corollary of Schaefer [29, page 80], $L(E, Y)$ becomes a locally convex topological vector space under $\sigma$-topology, where $Y$ is assumed a locally convex topological space.

Let $\operatorname{int} A$ and $\operatorname{Co} A$ denote the interior and convex hull of a set $A$, respectively. Let $C: X \rightarrow 2^{Y}$ be a set-valued mapping such that $C(x)$ is a closed pointed and convex cone with int $C(x) \neq \varnothing$ for each $x \in X$. Let $\eta: X \times X \rightarrow E$ and $H: X \times X \rightarrow Y$ be vector-valued mappings, $D: X \rightarrow 2^{X}$ and $T: X \rightarrow 2^{L(E, Y)}$ be two set-valued mappings, we introduced a new model of the generalized vector quasi-variational-like inequality, which is to find $\bar{x}$ in $X$ such that $\bar{x} \in D(\bar{x})$, and

$$
\forall y \in D(\bar{x}), \exists \hat{v} \in T(\bar{x}):\langle\hat{v}, \eta(y, \bar{x})\rangle+H(\bar{x}, y) \notin-\operatorname{int} C(\bar{x})
$$

Hindawi Publishing Corporation Journal of Inequalities and Applications Volume 2006, Article ID 59387, Pages 1-11 DOI 10.1155/JIA/2006/59387 
2 Generalized vector quasi-variational-like inequalities

It is easy to see that $\bar{x}$ is a solution of the problem (1.2) is equivalent to $\bar{x}$ in $X$ satisfying $\bar{x} \in D(\bar{x})$, and

$$
\forall y \in D(\bar{x}),\langle T(\bar{x}), \eta(y, \bar{x})\rangle+H(\bar{x}, y) \nsubseteq-\operatorname{int} C(\bar{x}),
$$

where $\langle T(\bar{x}), \eta(y, \bar{x})\rangle=\cup_{v \in T(\bar{x})}\langle v, \eta(y, \bar{x})\rangle$.

The following problems are the special cases of the problem (1.2).

(i) If $H(x, y) \equiv 0$ for all $x, y \in X$, then the problem (1.2) reduces to finding $\bar{x}$ in $X$ such that $\bar{x} \in D(\bar{x})$, and

$$
\forall y \in D(\bar{x}), \exists \hat{v} \in T(\bar{x}):\langle\hat{v}, \eta(y, \bar{x})\rangle \notin-\operatorname{int} C(\bar{x}) .
$$

This problem was also called generalized vector quasi-variational-like inequality and studied with certain monotonicity by Ding [13], and problem (1.4) contains as special cases the generalized vector variational-like inequality in $[1,2,14,15,28]$ and the generalized vector quasi-variational inequality studied by Chen and $\mathrm{Li}$ [10] and Lee et al. [22] and those vector variational inequalities in $[6-9,11,12,16,19-21,23,26,30,33-37]$.

(ii) If $T: X \rightarrow 2^{L(E, Y)}$ is a zero operator, then the problem (1.2) reduces to the vector quasi-equilibrium problem, which is to find $\bar{x}$ in $X$ such that $\bar{x} \in D(\bar{x})$, and

$$
H(\bar{x}, y) \notin-\operatorname{int} C(\bar{x}), \quad \forall y \in D(\bar{x}) .
$$

Problem (1.5) includes the vector equilibrium problem researched by many authors (see $[4,5,17,24,25,27])$.

In this paper, we establish existence results of solutions for both problem (1.2) and problem (1.4) with non-monotonicity and non-compactness. Our results extend and improve some main results of $[15,28]$.

In order to prove the main results, we need the following definitions and lemmas.

Definition 1.1 (see [15]). Let $E, Y$ be two real topological vector spaces, $X$ be a nonempty and convex subset of $E, C: X \rightarrow 2^{Y}$ be a set-valued mapping such that $C(x)$ is a closed pointed and convex cone with apex at 0 for each $x \in X$. Let $\eta: X \times X \rightarrow E$ be a singlevalued mapping. $T: X \rightarrow 2^{L(E, Y)}$ is said to satisfy the generalized $L-\eta$-condition if and only if for any finite set $\left\{y_{1}, y_{2}, \ldots, y_{n}\right\}$ in $X, \bar{x}=\sum_{j=1}^{n} \alpha_{j} y_{j}$ with $\alpha_{j} \geq 0$ and $\sum_{j=1}^{n} \alpha_{j}=1$, there exists $\bar{v} \in T(\bar{x})$, such that

$$
\left\langle\bar{v}, \sum_{j=1}^{n} \alpha_{j} \eta\left(y_{j}, \bar{x}\right)\right\rangle \notin-\operatorname{int} C(\bar{x}) .
$$

Remark 1.2. If $\eta(y, x)$ is affine in the first argument and $\forall x \in X, \exists v \in T(x)$, such that

$$
\langle\bar{v}, \eta(x, x)\rangle \notin-\operatorname{int} C(x),
$$

Then $T$ satisfies the generalized $L-\eta$-condition. 
If $\eta(y, x)=y-x, \forall x, y \in X$, then we have that

$$
\left\langle\bar{v}, \sum_{j=1}^{n} \alpha_{j}\left(y_{j}-\bar{x}\right)\right\rangle=\langle\bar{v}, \bar{x}-\bar{x}\rangle=0 \notin-\operatorname{int} C(\bar{x}), \quad \forall v \in T(\bar{x}),
$$

and hence $T$ satisfies the generalized $L-\eta$-condition trivially.

Definition 1.3 (see [32]). Let $X$ and $Y$ be two topological spaces and $T: X \rightarrow 2^{Y}$ be a set-valued mapping. Then

(1) $T$ is said to be upper semicontinuous if, for any $x_{0} \in X$ and for each open set $U$ in $Y$ containing $T\left(x_{0}\right)$, there is a neighbourhood $V$ of $x_{0}$ in $X$ such that $T(x) \subseteq U$, for all $x \in V$.

(2) $T$ is said to have open lower sections if the set $T^{-1}(y)=\{x \in X: y \in T(x)\}$ is open in $X$ for each $y \in Y$.

(3) $T$ is said to be closed, if the set $\{(x, y) \in X \times Y: y \in T(x)\}$ is closed in $X \times Y$.

Definition 1.4. Let $C: X \rightarrow 2^{Y}$ be a set-valued mapping. $H: X \times X \rightarrow Y$ is said to be $0-C(x)$ diagonally convex with respect to the second argument if, for any finite subset $\left\{y_{1}, y_{2}, \ldots, y_{n}\right\}$ in $X$, and any $x \in X$ with $x=\sum_{j=1}^{n} \alpha_{j} y_{j}\left(\alpha_{j} \geq 0, \sum_{j=1}^{n} \alpha_{j}=1\right)$, we have

$$
\sum_{j=1}^{n} \alpha_{j} H\left(\bar{x}, y_{j}\right) \in C(\bar{x})
$$

$H: X \times X \rightarrow Y$ is said to be $0-C(x)$ diagonally concave with respect to the second argument if $-H$ is $0-C(x)$ diagonally convex with respect to the second argument.

Remark 1.5. If $Y=R \cup\{ \pm \infty\}$ and $C(x)=\{r \in R: r \geq 0\}$, then the $0-C(x)$ diagonal concavity of $H$ reduces to the 0 -diagonal concavity of $H$ in [38].

Lemma 1.6 (see [32]). Let $X$ and $Y$ be two topological spaces. Suppose $T: X \rightarrow 2^{Y}$ and $K$ : $X \rightarrow 2^{Y}$ are set-valued mappings having open lower sections, then (i) the set-valued mapping $F: X \rightarrow 2^{Y}$ defined by, for each $x \in X, F(x)=\operatorname{Co}(T(x))$ has open lower sections. (ii) the setvalued mapping $\theta: X \rightarrow 2^{Y}$ defined by, for each $x \in X, \theta(x)=T(x) \cap K(x)$ has open lower sections.

Lemma 1.7 (see [3]). Let $X$ and $Y$ be topological spaces. If $T: X \rightarrow 2^{Y}$ is an upper semicontinuous set-valued mapping with closed values, then $T$ is closed.

Lemma 1.8 (see [31]). Let $X$ and $Y$ be topological spaces and $T: X \rightarrow 2^{Y}$ be an upper semicontinuous set-valued mapping with compact values. Suppose $\left\{x_{\alpha}\right\}$ is a net in $X$ such that $x_{\alpha} \rightarrow x_{0}$. If $y_{\alpha} \in T\left(x_{\alpha}\right)$ for each $\alpha$, then there is a $y_{0} \in T\left(x_{0}\right)$ and a subset $\left\{y_{\beta}\right\}$ of $\left\{y_{\alpha}\right\}$ such that $y_{\beta} \rightarrow y_{0}$.

Lemma 1.9 (see [18]). Let $X$ be a nonempty convex subset of a Hausdorff topological vector space $E$ and $S: X \rightarrow 2^{X}$ be a set-valued mapping such that for each $x \in X, x \notin \operatorname{Co}(S(x))$ and for each $y \in X, S^{-1}(y)$ is open in $X$. Suppose further that there exist a nonempty compact 
4 Generalized vector quasi-variational-like inequalities

subset $N$ of $X$ and a nonempty compact convex subset $B$ of $X$ such that $\operatorname{Co}(S(x)) \cap B \neq \varnothing$ for all $x \in X \backslash N$.

Then there exists a point $\bar{x} \in X$ such that $S(\bar{x})=\varnothing$.

\section{Main results}

In this section, we will present some existence results of solutions for the two types of generalized vector quasi-variational inequalities without monotonicity and compactness.

Theorem 2.1. Let $Y$ be a real Hausdorff topological vector space, $X$ be a nonempty and convex set in a real locally convex Hausdorff topological vector space $E$, and $L(E, Y)$ be equipped with the $\sigma$-topology. Let $D: X \rightarrow 2^{X}$ be a set-valued mapping such that $\forall x \in X, D(x)$ is nonempty and convex, $D^{-1}(y)$ is open in $X, \forall y \in X$, and the set $W=\{x \in X: x \in D(x)\}$ is closed in $X$. Let $C: X \rightarrow 2^{Y}$ be a set-valued mapping such that $C(x)$ is a closed, pointed and convex cone with int $C(x) \neq \varnothing$ for each $x \in X$. Assume that the following conditions are satisfied.

(i) The set-valued mapping $M=Y \backslash(-\operatorname{int} C): X \rightarrow 2^{Y}$ is upper semicontinuous on $X$.

(ii) The set-valued mapping $T: X \rightarrow 2^{L(E, Y)}$ is upper semicontinuous on $X$ with compact values and $\eta: X \times X \rightarrow E$ is continuous with respect to the second argument, such that $T$ satisfies the generalized $L-\eta$-condition.

(iii) $H: X \times X \rightarrow Y$ is continuous with respect to the first argument and $0-C(x)$ diagonally convex with respect to the second argument.

(iv) There exist a nonempty and compact subset $N$ of $X$ and a nonempty, compact and convex subset $B$ of $X$ such that $\forall x \in X \backslash N, \exists \bar{y} \in B$, such that $\bar{y} \in D(x)$ and $\langle v, \eta(\bar{y}, x)\rangle+H(x, \bar{y}) \in-\operatorname{int} C(x), \forall v \in T(x)$.

Then, there exists a point $\bar{x} \in X$ such that $\bar{x} \in D(\bar{x})$, and

$$
\forall y \in D(\bar{x}), \exists \hat{v} \in T(\bar{x}):\langle\hat{v}, \eta(y, \bar{x})\rangle+H(\bar{x}, y) \notin-\operatorname{int} C(\bar{x}) .
$$

Proof. Define a set-valued mapping $P: X \rightarrow 2^{X}$ by

$$
\begin{aligned}
P(x) & =\{y \in X:\langle T(x), \eta(y, x)\rangle+H(x, y) \subseteq-\operatorname{int} C(x)\} \\
& =\{y \in X:\langle v, \eta(y, x)\rangle+H(x, y) \in-\operatorname{int} C(x), \forall v \in T(x)\}, \quad \forall x \in X .
\end{aligned}
$$

We first prove that $x \notin \operatorname{Co} P(x)$ for all $x \in X$. To see this, suppose, by way of contradiction, that there exists some point $\bar{x} \in X$ such that $\bar{x} \in \operatorname{Co}(P(\bar{x}))$. Then there exists finite points $y_{1}, y_{2}, \ldots, y_{n}$ in $X$, and $\alpha_{j} \geq 0$ with $\sum_{j=1}^{n} \alpha_{j}=1$ such that $\bar{x}=\sum_{j=1}^{n} \alpha_{j} y_{j}$ and $y_{j} \in P(\bar{x})$ for all $j=1,2, \ldots, n$. That is,

$$
\left\langle v, \eta\left(y_{j}, \bar{x}\right)\right\rangle+H\left(\bar{x}, y_{j}\right) \in-\operatorname{int} C(\bar{x}), \quad \forall v \in T(x), j=1,2, \ldots, n .
$$

Since int $C(\bar{x})$ is a convex set, we obtain

$$
\left\langle v, \sum_{j=1}^{n} \alpha_{j} \eta\left(y_{j}, \bar{x}\right)\right\rangle+\sum_{j=1}^{n} \alpha_{j} H\left(\bar{x}, y_{j}\right) \in-\operatorname{int} C(\bar{x}), \quad \forall v \in T(x) .
$$


From the $0-C(x)$ diagonal convexity with respect to the second argument of $H$, we have

$$
\sum_{j=1}^{n} \alpha_{j} H\left(\bar{x}, y_{j}\right) \in C(\bar{x})
$$

By (2.4) and (2.5), we get, for all $v \in T(\bar{x})$,

$$
\left\langle v, \sum_{j=1}^{n} \alpha_{j} \eta\left(y_{j}, \bar{x}\right)\right\rangle \in-\sum_{j=1}^{n} \alpha_{j} H\left(\bar{x}, y_{j}\right)-\operatorname{int} C(\bar{x}) \subseteq-C(\bar{x})-\operatorname{int} C(\bar{x}) \subseteq-\operatorname{int} C(\bar{x}),
$$

which contradicts the fact that $T$ satisfies the generalized $L-\eta$-condition. Therefore $x \notin$ Co $P(x)$ for all $x \in X$.

Now we prove that the set

$$
\begin{aligned}
P^{-1}(y) & =\{x \in X:\langle T(x), \eta(y, x)\rangle+H(x, y) \subseteq-\operatorname{int} C(x)\} \\
& =\{x \in X:\langle v, \eta(y, x)\rangle+H(x, y) \in-\operatorname{int} C(x), \forall v \in T(x)\}
\end{aligned}
$$

is open for each $y \in X$. That is, $P$ has open lower sections in $X$. Consider the set-valued mapping $Q: X \rightarrow 2^{X}$ defined by

$$
\begin{aligned}
Q(y) & =\{x \in X:\langle T(x), \eta(y, x)\rangle+H(x, y) \notin-\operatorname{int} C(x)\} \\
& =\{x \in X: \exists v \in T(x) \text { such that }\langle v, \eta(y, x)\rangle+H(x, y) \notin-\operatorname{int} C(x)\} .
\end{aligned}
$$

We only need to prove that $Q(y)$ is closed for all $y \in X$. In fact, consider a net $x_{t} \in Q(y)$ such that $x_{t} \rightarrow x \in X$. Since $x_{t} \in Q(y)$, there exists $s_{t} \in T\left(x_{t}\right)$ such that

$$
\left\langle s_{t}, \eta\left(y, x_{t}\right)\right\rangle+H\left(x_{t}, y\right) \notin-\operatorname{int} C\left(x_{t}\right) .
$$

From the upper semicontinuity and compact values of $T$ and Lemma 1.8, it suffices to find a subset $\left\{s_{t_{j}}\right\}$ which converges to some $s \in T(x)$. By [15, Lemma 1, page 114], we know that $\langle\cdot\rangle$ is continuous, and hence

$$
\left\langle s_{t_{j}}, \eta\left(y, x_{t_{j}}\right)\right\rangle+H\left(x_{t_{j}}, y\right) \longrightarrow\langle s, \eta(y, x)\rangle+H(x, y) .
$$

By Lemma 1.7 and upper semicontinuity of $M$, we have $\langle s, \eta(y, x)\rangle+H(x, y) \notin-\operatorname{int} C(x)$, and hence $x \in Q(y), Q(y)$ is closed. Therefore, $P$ has open lower sections in $X$, and by Lemma 1.6, we know that $\operatorname{Co} P: X \rightarrow 2^{X}$ also has open lower sections. Also define another set-valued mapping $S: X \rightarrow 2^{X}$ by

$$
S(x)= \begin{cases}D(x) \cap \operatorname{Co} P(x) & \text { if } x \in W \\ D(x) & \text { if } x \notin W .\end{cases}
$$


6 Generalized vector quasi-variational-like inequalities

Then, it is clear that $\forall x \in X, S(x)$ is convex, and $x \notin S(x)=\operatorname{Co} S(x)$. Since $\forall y \in X$,

$$
\begin{aligned}
S^{-1}(y) & =\{x \in X: y \in S(x)\} \\
& =\{x \in W: y \in D(x) \cap \operatorname{Co} P(x)\} \cup\{x \in X \backslash W: y \in D(x)\} \\
& =\left(W \cap D^{-1}(y) \cap \operatorname{Co} P^{-1}(y)\right) \cup\left[(X \backslash W) \cap D^{-1}(y)\right] \\
& =\left[\left(W \cap D^{-1}(y) \cap \operatorname{Co} P^{-1}(y)\right) \cup(X \backslash W)\right] \cap\left[\left(W \cap D^{-1}(y) \cap \operatorname{Co} P^{-1}(y)\right) \cup D^{-1}(y)\right] \\
& =\left\{X \cap\left[\left(D^{-1}(y) \cap \operatorname{Co} P^{-1}(y)\right) \cup(X \backslash W)\right]\right\} \cap\left[\left(W \cup D^{-1}(y)\right) \cap\left(D^{-1}(y)\right)\right] \\
& =\left[\left(D^{-1}(y) \cap \operatorname{Co} P^{-1}(y)\right) \cup(X \backslash W)\right] \cap D^{-1}(y) \\
& =\left(D^{-1}(y) \cap\left(\operatorname{Co} P^{-1}(y)\right)\right) \cup\left((X \backslash W) \cap\left(D^{-1}(y)\right)\right),
\end{aligned}
$$

and $D^{-1}(y), \operatorname{Co} P^{-1}(y)$ and $X \backslash W$ are open in $X$, we have $S^{-1}(y)$ is open in $X$.

Condition (iii) implies that there exist a nonempty compact subset $N$ of $X$ and a nonempty compact convex subset $B$ of $X$ such that $S(x) \cap B=\operatorname{Co} S(x) \cap B \neq \varnothing$ for all $x \in X \backslash N$. Hence, by Lemma 1.9, $\exists \bar{x} \in X$ such that $S(\bar{x})=\varnothing$. Since $\forall x \in X, D(x)$ is nonempty, we have $\bar{x} \in W$, and $D(\bar{x}) \cap \operatorname{Co} P(\bar{x})=\varnothing$. This implies $\bar{x} \in D(\bar{x})$ and $D(\bar{x}) \cap$ $P(\bar{x})=\varnothing$. Consequently, $\bar{x} \in D(\bar{x})$, and $\forall y \in D(\bar{x}), \exists v \in T(\bar{x})$ satisfying $\langle v, \eta(y, \bar{x})\rangle+$ $H(\bar{x}, y) \notin-\operatorname{int} C(\bar{x})$.

By Theorem 2.1 and Remark 1.2, we have the following corollary.

Corollary 2.2. Let $Y$ be a real Hausdorff topological vector space, $X$ be a nonempty and convex set in a real locally convex Hausdorff topological vector space $E$, and $L(E, Y)$ be equipped with the $\sigma$-topology. Let $D: X \rightarrow 2^{X}$ be a set-valued mapping such that $\forall x \in X$, $D(x)$ is nonempty and convex, $D^{-1}(y)$ is open in $X, \forall y \in X$, and the set $W=\{x \in X: x \in$ $D(x)\}$ is closed in $X$. Let $C: X \rightarrow 2^{Y}$ be a set-valued mapping such that $C(x)$ is a closed, pointed and convex cone with int $C(x) \neq \varnothing$ for each $x \in X$. Assume that the following conditions are satisfied.

(i) The set-valued mapping $M=Y \backslash(-\operatorname{int} C): X \rightarrow 2^{Y}$ is upper semicontinuous on $X$.

(ii) The set-valued mapping $T: X \rightarrow 2^{L(E, Y)}$ is upper semicontinuous on $X$ with compact values and $\eta: X \times X \rightarrow E$ is continuous with respect to the second argument and affine with respect to the first argument such that $\forall x \in X, \exists v \in T(x)$, satisfying $\langle\bar{v}, \eta(x, x)\rangle \notin-\operatorname{int} C(x)$.

(iii) $H: X \times X \rightarrow Y$ is continuous with respect to the first argument and $0-C(x)$ diagonally convex with respect to the second argument.

(iv) There exist a nonempty and compact subset $N$ of $X$ and a nonempty, compact and convex subset $B$ of $X$ such that $\forall x \in X \backslash N, \exists \bar{y} \in B$, such that $\bar{y} \in D(x)$ and $\langle v, \eta(\bar{y}, x)\rangle+H(x, \bar{y}) \in-\operatorname{int} C(x), \forall v \in T(x)$.

Then, there exists a point $\bar{x} \in X$ such that $\bar{x} \in D(\bar{x})$, and

$$
\forall y \in D(\bar{x}), \exists \hat{v} \in T(\bar{x}):\langle\hat{v}, \eta(y, \bar{x})\rangle+H(\bar{x}, y) \notin-\operatorname{int} C(\bar{x})
$$


If $H(x, x)=0 \forall x \in X$, then by Theorem 2.1 and Corollary 2.2, we have the following corollary.

Corollary 2.3. Let $Y$ be a real Hausdorff topological vector space, $X$ be a nonempty and convex set in a real locally convex Hausdorff topological vector space $E$, and $L(E, Y)$ be equipped with the $\sigma$-topology. Let $D: X \rightarrow 2^{X}$ be a set-valued mapping such that $\forall x \in X$, $D(x)$ is nonempty and convex, $D^{-1}(y)$ is open in $X, \forall y \in X$, and the set $W=\{x \in X: x \in$ $D(x)\}$ is closed in $X$. Let $C: X \rightarrow 2^{Y}$ be a set-valued mapping such that $C(x)$ is a closed, pointed and convex cone with int $C(x) \neq \varnothing$ for each $x \in X$. Assume that the following conditions are satisfied.

(i) The set-valued mapping $M=Y \backslash(-\operatorname{int} C): X \rightarrow 2^{Y}$ is upper semicontinuous on $X$.

(ii) There exist a nonempty and compact subset $N$ of $X$ and a nonempty, compact and convex subset $B$ of $X$ such that $\forall x \in X \backslash N, \exists \bar{y} \in B$, such that $\bar{y} \in D(x)$ and $\langle v, \eta(\bar{y}, x)\rangle \in-\operatorname{int} C(x), \forall v \in T(x)$.

(iii) The set-valued mapping $T: X \rightarrow 2^{L(E, Y)}$ is upper semicontinuous on $X$ with compact values and $\eta: X \times X \rightarrow E$ is continuous with respect to the second argument. Moreover, one of the following conditions satisfied

(iv) $T: X \rightarrow 2^{L(E, Y)}$ satisfies the generalized $L-\eta$-condition.

$\mathrm{Or}$

(v) $\eta: X \times X \rightarrow E$ is affine with respect to the first argument such that $\forall x \in X, \exists v \in$ $T(x)$, satisfying $\langle\bar{v}, \eta(x, x)\rangle \notin-\operatorname{int} C(x)$.

Then, there exists a point $\bar{x} \in X$ such that $\bar{x} \in D(\bar{x})$, and

$$
\forall y \in D(\bar{x}), \exists \hat{v} \in T(\bar{x}):\langle\hat{v}, \eta(y, \bar{x})\rangle \notin-\operatorname{int} C(\bar{x}) .
$$

Remark 2.4. Theorem 2.1, Corollaries 2.2 and 2.3 extend and improve [15, Theorem 1 and Corollary 1] and [28, Theorem 1] without monotonicity and compactness.

If $T$ is a zero operator, then by Theorem 2.1, we have the following corollary.

Corollary 2.5. Let $Y$ be a real Hausdorff topological vector space, $X$ be a nonempty and convex set in a real locally convex Hausdorff topological vector space $E$. Let $D: X \rightarrow 2^{X}$ be a set-valued mapping such that $\forall x \in X, D(x)$ is nonempty and convex, $D^{-1}(y)$ is open in $X$, $\forall y \in X$, and the set $W=\{x \in X: x \in D(x)\}$ is closed in $X$. Let $C: X \rightarrow 2^{Y}$ be a set-valued mapping such that $C(x)$ is a closed, pointed and convex cone with int $C(x) \neq \varnothing$ for each $x \in X$. Assume that the following conditions are satisfied.

(i) The set-valued mapping $M=Y \backslash(-\operatorname{int} C): X \rightarrow 2^{Y}$ is upper semicontinuous on $X$.

(ii) $H: X \times X \rightarrow Y$ is continuous with respect to the first argument and $0-C(x)$ diagonally convex with respect to the second argument.

(iii) There exist a nonempty and compact subset $N$ of $X$ and a nonempty, compact and convex subset $B$ of $X$ such that $\forall x \in X \backslash N, \exists \bar{y} \in B$, such that $\bar{y} \in D(x)$ and $H(x, \bar{y}) \in-\operatorname{int} C(x)$.

Then, there exists a point $\bar{x} \in X$ such that $\bar{x} \in D(\bar{x})$, and $H(\bar{x}, y) \notin-\operatorname{int} C(\bar{x}), \forall y \in D(\bar{x})$.

Theorem 2.6. Let $Y$ be a real Hausdorff topological vector space, $X$ be a nonempty and convex set in a real locally convex Hausdorff topological vector space $E$, and $L(E, Y)$ be equipped with the $\sigma$-topology. Let $D: X \rightarrow 2^{X}$ be a set-valued mapping such that $\forall x \in X, D(x)$ is 
nonempty and convex, $D^{-1}(y)$ is open in $X, \forall y \in X$, and the set $W=\{x \in X: x \in D(x)\}$ is closed in $X$. Let $C: X \rightarrow 2^{Y}$ be a set-valued mapping such that $C(x)$ is a closed, pointed and convex cone with int $C(x) \neq \varnothing$ for each $x \in X$. Assume that the following conditions are satisfied.

(i) The set-valued mapping $M=Y \backslash(-\operatorname{int} C): X \rightarrow 2^{Y}$ is upper semicontinuous on $X$.

(ii) The set-valued mapping $T: X \rightarrow 2^{L(E, Y)}$ is upper semicontinuous on $X$ with compact values and $\eta: X \times X \rightarrow E$ is continuous with respect to the second argument, and there exists a mapping $h: X \times X \rightarrow Y$, such that:

(a) $\forall x, y \in X, \exists v \in T(x)$, such that

$$
h(x, y)-\langle v, \eta(y, x)\rangle \in-\operatorname{int} C(x) .
$$

(b) For any finite set $\left\{y_{1}, y_{2}, \ldots, y_{n}\right\} \subseteq X$ and $\bar{x}=\sum_{j=1}^{n} \alpha_{j} y_{j}$ with $\alpha_{j} \geq 0$ and $\sum_{j=1}^{n} \alpha_{j}=1$, there is a $j \in\{1,2, \ldots, n\}$, such that $h\left(\bar{x}, y_{j}\right) \notin-\operatorname{int} C(\bar{x})$.

(iii) There exist a nonempty and compact subset $N$ of $X$ and a nonempty, compact and convex subset $B$ of $X$ such that $\forall x \in X \backslash N, \exists \bar{y} \in B$, such that $\bar{y} \in D(x)$ and

$$
\langle v, \eta(\bar{y}, x)\rangle \in-\operatorname{int} C(x), \quad \forall v \in T(x) .
$$

Then, there exists a point $\bar{x} \in X$ such that $\bar{x} \in D(\bar{x})$, and

$$
\forall y \in D(\bar{x}), \exists \hat{v} \in T(\bar{x}):\langle\hat{v}, \eta(y, \bar{x})\rangle \notin-\operatorname{int} C(\bar{x}) .
$$

Proof. Define two set-valued mappings $P: X \rightarrow 2^{X}, P_{1}: X \rightarrow 2^{X}$ by

$$
\begin{gathered}
P(x)=\{y \in X:\langle v, \eta(y, x)\rangle \in-\operatorname{int} C(x), \forall v \in T(x)\}, \quad \forall x \in X . \\
P_{1}(x)=\{y \in X: h(x, y) \in-\operatorname{int} C(x)\}, \quad \forall x \in X .
\end{gathered}
$$

We first prove that $x \notin \operatorname{Co}\left(P_{1}(x)\right)$ for all $x \in X$. To see this, suppose, by way of contradiction, that there exists some point $\bar{x} \in X$ such that $\bar{x} \in \operatorname{Co}\left(P_{1}(\bar{x})\right)$. Then there exists finite points $y_{1}, y_{2}, \ldots, y_{n}$ in $X$, and $\alpha_{j} \geq 0$ with $\sum_{j=1}^{n} \alpha_{j}=1$ such that $\bar{x}=\sum_{j=1}^{n} \alpha_{j} y_{j}$ and $y_{j} \in P_{1}(\bar{x})$ for all $j=1,2, \ldots, n$. That is,

$$
h\left(\bar{x}, y_{j}\right) \in-\operatorname{int} C(\bar{x}), \quad j=1,2, \ldots, n .
$$

This contradicts to the condition (ii)(b). Therefore $x \notin \operatorname{Co}\left(P_{1}(x)\right)$ for all $x \in X$.

The condition (ii)(a) implies that $P_{1}(x) \supseteq P(x)$ for all $x \in X$. Hence, $x \notin \operatorname{Co}(P(x))$, $\forall x \in X$.

The remainder of the proof is similar to that in the proof of Theorem 2.1.

Corollary 2.7. Let $Y$ be a real Hausdorff topological vector space, $X$ be a nonempty and convex set in a real locally convex Hausdorff topological vector space $E$, and $L(E, Y)$ be equipped with the $\sigma$-topology. Let $D: X \rightarrow 2^{X}$ be a set-valued mapping such that $\forall x \in X$, $D(x)$ is nonempty and convex, $D^{-1}(y)$ is open in $X, \forall y \in X$, and the set $W=\{x \in X: x \in$ $D(x)\}$ is closed in $X$. Let $C: X \rightarrow 2^{Y}$ be a set-valued mapping such that $C(x)$ is a closed, 
pointed and convex cone with int $C(x) \neq \varnothing$ for each $x \in X$. Assume that the following conditions are satisfied.

(i) The set-valued mapping $M=Y \backslash(-\operatorname{int} C): X \rightarrow 2^{Y}$ is upper semicontinuous on $X$.

(ii) The set-valued mapping $T: X \rightarrow 2^{L(E, Y)}$ is upper semicontinuous on $X$ with compact values and $\eta: X \times X \rightarrow E$ is continuous with respect to the second argument, and there exists a mapping $h: X \times X \rightarrow Y$, such that:

(a) $\forall x, y \in X, \exists v \in T(x)$, such that

$$
h(x, y)-\langle v, \eta(y, x)\rangle \in-\operatorname{int} C(x)
$$

(b) the set $\{y \in X: h(x, y) \in-\operatorname{int} C(x)\}$ is convex for all $x \in X$;

(c) $h(x, x) \notin-\operatorname{int} C(x), \forall x \in X$.

Then, there exists $\bar{x} \in X$, such that $\bar{x} \in D(\bar{x})$ and $\langle T(\bar{x}), \eta(y, \bar{x})\rangle \notin-\operatorname{int} C(\bar{x}), \forall y$ $\in D(\bar{x})$.

(iii) There exist a nonempty and compact subset $N$ of $X$ and a nonempty, compact and convex subset $B$ of $X$ such that $\forall x \in X \backslash N, \exists \bar{y} \in B$, such that $\bar{y} \in D(x)$ and $\langle v, \eta(\bar{y}, x)\rangle \in-\operatorname{int} C(x), \forall v \in T(x)$.

Then, there exists a point $\bar{x} \in X$ such that $\bar{x} \in D(\bar{x})$, and

$$
\forall y \in D(\bar{x}), \exists \hat{v} \in T(\bar{x}):\langle\hat{v}, \eta(y, \bar{x})\rangle \notin-\operatorname{int} C(\bar{x}) .
$$

Proof. Following the same argument of the proof of [15, Corollary 3], by the condition (ii)(b) and (ii)(c), we know that the condition (ii)(b) of Theorem 2.6 holds. By Theorem 2.6, we know that the conclusion is correct.

Remark 2.8. Theorem 2.6 and Corollary 2.7, respectively, extend and improve [15, Theorem 2 and Corollary 3].

\section{Acknowledgments}

The authors would like to express their thanks to the referees for helpful suggestions. This paper was supported by the National Natural Science Foundation of China (Grant No. 10171118) and Education Committee Project Research Foundation of Chongqing (Grant No. 030801) and the Science Committee Project Research Foundation of Chongqing (Grant No. 8409).

\section{References}

[1] Q. H. Ansari, A note on generalized vector variational-like inequalities, Optimization 41 (1997), no. 3, 197-205.

[2] _ Extended generalized vector variational-like inequalities for nonmonotone multivalued maps, Annales des Sciences Mathématiques du Québec 21 (1997), no. 1, 1-11.

[3] J.-P. Aubin and I. Ekeland, Applied Nonlinear Analysis, Pure and Applied Mathematics (New York), John Wiley \& Sons, New York, 1984.

[4] M. Bianchi, N. Hadjisavvas, and S. Schaible, Vector equilibrium problems with generalized monotone bifunctions, Journal of Optimization Theory and Applications 92 (1997), no. 3, 527-542.

[5] O. Chadli, Y. Chiang, and S. Huang, Topological pseudomonotonicity and vector equilibrium problems, Journal of Mathematical Analysis and Applications 270 (2002), no. 2, 435-450. 
[6] G. Y. Chen, Existence of solutions for a vector variational inequality: an extension of the HartmannStampacchia theorem, Journal of Optimization Theory and Applications 74 (1992), no. 3, 445456.

[7] G. Y. Chen and G. M. Cheng, Vector Variational Inequalities and Vector Optimization, Lecture Notes in Economics and Mathematical Systems, vol. 285, Springer, Berlin, 1987.

[8] G. Y. Chen and B. D. Craven, Approximate dual and approximate vector variational inequality for multiobjective optimization, Australian Mathematical Society. Journal. Series A. 47 (1989), no. 3 , 418-423.

[9] _ A vector variational inequality and optimization over an efficient set, Zeitschrift für Operations Research 34 (1990), no. 1, 1-12.

[10] G. Y. Chen and S. J. Li, Existence of solutions for a generalized vector quasivariational inequality, Journal of Optimization Theory and Applications 90 (1996), no. 2, 321-334.

[11] G. Y. Chen and X. Q. Yang, The vector complementary problem and its equivalences with the weak minimal element in ordered spaces, Journal of Mathematical Analysis and Applications $\mathbf{1 5 3}$ (1990), no. 1, 136-158.

[12] A. Daniilidis and N. Hadjisavvas, Existence theorems for vector variational inequalities, Bulletin of the Australian Mathematical Society 54 (1996), no. 3, 473-481.

[13] X. P. Ding, The generalized vector quasi-variational-like inequalities, Computers \& Mathematics with Applications 37 (1999), no. 6, 57-67.

[14] X. P. Ding and E. Tarafdar, Generalized vector variational-like inequalities with $C_{x}-\eta$ pseudomonotone set-valued mappings, Vector Variational Inequalities and Vector Equilibria (F. Giannessi, ed.), Nonconvex Optim. Appl., vol. 38, Kluwer Academic, Dordrecht, 2000, pp. 125140 .

[15] _ Generalized vector variational-like inequalities without monotonicity, Vector Variational Inequalities and Vector Equilibria (F. Giannessi, ed.), Nonconvex Optim. Appl., vol. 38, Kluwer Academic, Dordrecht, 2000, pp. 113-124.

[16] F. Giannessi, Theorems of alternative, quadratic programs and complementarity problems, Variational Inequalities and Complementarity Problems (Proc. Internat. School, Erice, 1978) (R. W. Cottle, F. Giannessi, and J.-L. Lions, eds.), John Wiley \& Sons, Chichester, 1980, pp. 151-186.

[17] N. Hadjisavvas and S. Schaible, From scalar to vector equilibrium problems in the quasimonotone case, Journal of Optimization Theory and Applications 96 (1998), no. 2, 297-309.

[18] W. K. Kim, Existence of maximal element and equilibrium for a nonparacompact $N$-person game, Proceedings of the American Mathematical Society 116 (1992), no. 3, 797-807.

[19] I. V. Konnov and J. C. Yao, On the generalized vector variational inequality problem, Journal of Optimization Theory and Applications 206 (1997), no. 1, 42-58.

[20] G. M. Lee, D. S. Kim, and B. S. Lee, Generalized vector variational inequality, Applied Mathematics Letters 9 (1996), no. 1, 39-42.

[21] G. M. Lee, D. S. Kim, B. S. Lee, and S. J. Cho, Generalized vector variational inequality and fuzzy extension, Applied Mathematics Letters 6 (1993), no. 6, 47-51.

[22] G. M. Lee, B. S. Lee, and S.-S. Chang, On vector quasivariational inequalities, Journal of Mathematical Analysis and Applications 203 (1996), no. 3, 626-638.

[23] K. L. Lin, D.-P. Yang, and J. C. Yao, Generalized vector variational inequalities, Journal of Optimization Theory and Applications 92 (1997), no. 1, 117-125.

[24] L. J. Lin, Z. T. Yu, and G. Kassay, Existence of equilibria for multivalued mappings and its application to vectorial equilibria, Journal of Optimization Theory and Applications 114 (2002), no. 1, 189-208.

[25] W. Oettli, A remark on vector-valued equilibria and generalized monotonicity, Acta Mathematica Vietnamica 22 (1997), no. 1, 213-221.

[26] W. Oettli and D. Schläger, Existence of equilibria for monotone multivalued mappings, Mathematical Methods of Operations Research 48 (1998), no. 2, 219-228. 
[27] J. W. Peng, Equilibrium problems for W-spaces, Mathematica Applicata (Wuhan) 12 (1999), no. 3, 81-87 (Chinese).

[28] L. Qun, Generalized vector variational-like inequalities, Vector Variational Inequalities and Vector Equilibria (F. Giannessi, ed.), Nonconvex Optim. Appl., vol. 38, Kluwer Academic, Dordrecht, 2000, pp. 353-369.

[29] H. H. Schaefer, Topological vector spaces, Graduate Texts in Mathematics, vol. 3, Springer, New York, 1971.

[30] A. H. Siddiqi, Q. H. Ansari, and A. Khaliq, On vector variational inequalities, Journal of Optimization Theory and Applications 84 (1995), no. 1, 171-180.

[31] C. H. Su and V. M. Sehgal, Some fixed point theorems for condensing multifunctions in locally convex spaces, Proceedings of the American Mathematical Society 50 (1975), 150-154.

[32] G. Q. Tian and J. Zhou, Quasi-variational inequalities without the concavity assumption, Journal of Mathematical Analysis and Applications 172 (1993), no. 1, 289-299.

[33] X. Q. Yang, Generalized convex functions and vector variational inequalities, Journal of Optimization Theory and Applications 79 (1993), no. 3, 563-580.

[34] - Vector complementarity and minimal element problems, Journal of Optimization Theory and Applications 77 (1993), no. 3, 483-495.

[35] _ _ Vector variational inequality and its duality, Nonlinear Analysis 21 (1993), no. 11, 869877.

[36] X. Q. Yang and J. C. Yao, Gap functions and existence of solutions to set-valued vector variational inequalities, Journal of Optimization Theory and Applications 115 (2002), no. 2, 407-417.

[37] S. J. Yu and J. C. Yao, On vector variational inequalities, Journal of Optimization Theory and Applications 89 (1996), no. 3, 749-769.

[38] J. Zhou and G. Chen, Diagonal convexity conditions for problems in convex analysis and quasivariational inequalities, Journal of Mathematical Analysis and Applications 132 (1988), no. 1, 213-225.

Jian-Wen Peng: College of Mathematics and Computer Science, Chongqing Normal University, Chongqing 400047, China

Current address: Department of Mathematics, Inner Mongolia University, Hohhot 010021, China

E-mail address: jwpeng6@yahoo.com.cn

Xin-Min Yang: College of Mathematics and Computer Science, Chongqing Normal University, Chongqing 400047, China 